\title{
Portable Gait Lab: Instantaneous centre of mass velocity using three inertial measurement units
}

\author{
Mohamed Irfan Mohamed Refai*, Bert-Jan F. van Beijnum*, Jaap H. Buurke†,*, and Peter H. Veltink* \\ Email:m.i.mohamedrefai, b.j.f.vanbeijnum@utwente.nl; j.buurke@rrd.nl; p.h.veltink@utwente.nl \\ *Biomedical Signals and Systems, University of Twente, NL \\ $\dagger$ Roessingh Research and Development, Enschede, NL
}

\begin{abstract}
Estimating instantaneous 3D Centre of Mass velocity (VCOM) using wearables can improve ambulatory gait monitoring. Inertial Measurement Units (IMU) are commonly used to estimate VCOM, although, studies have either measured only the magnitude, or use machine learning methods. Here, we propose a three IMU setup, where the VCOM is obtained by a complementary filter method. This method fuses high frequency information achieved using strapdown integration of accelerations measured at the pelvis with low frequency information of VCOM obtained from foot velocities. This method is applied in variable gait which includes turns. The root mean square of the error between the IMU estimated VCOM against a reference VICON measurement was found to be $0.1 \pm 0.02 \mathrm{~m} / \mathrm{s}$ across all walking tasks. This method provides a drift free ambulatory estimation of CoM velocity using minimal IMUs.
\end{abstract}

\section{INTRODUCTION}

Estimation of the centre of mass (CoM) velocity (VCOM) has several practical applications, including measuring gait parameters, and balance measures such as extrapolated CoM (XCOM) [1]. Inertial Measurement units (IMUs) offer solutions for ambulatory estimation of VCOM [2], [3]. However, so far, studies have either measured the magnitude of VCOM [2], or used machine learning techniques [3] which require additional training.

Here, we propose a setup of three IMUs for estimating the VCOM; one IMU at the pelvis, and one on each foot. Information about VCOM is extracted from the movement of the pelvis and feet, and are fused using a complementary filter method [3], [4], resulting in drift free instantaneous estimation of 3D VCOM.

The following sections describe the methods used to obtain the instantaneous 3D VCOM in a special current step frame $\left(\psi_{c s}\right)$ [5], and describes the performance of the method in variable overground gait.

\section{Methods}

First, Section II-A provides a brief overview of reference frames used in this study [5]. Cyclical changes in VCOM was obtained by integrating pelvis accelerations and high pass filtering the output [2], [3]. Furthermore, average movement of the feet encode information about the VCOM. These two sources of VCOM can be fused using a complementary filter method. Section II-B shows the method used for strapdown integration of ACOM. Here we assume that gait is modelled as an inverted pendulum, with the pelvis IMU accelerations

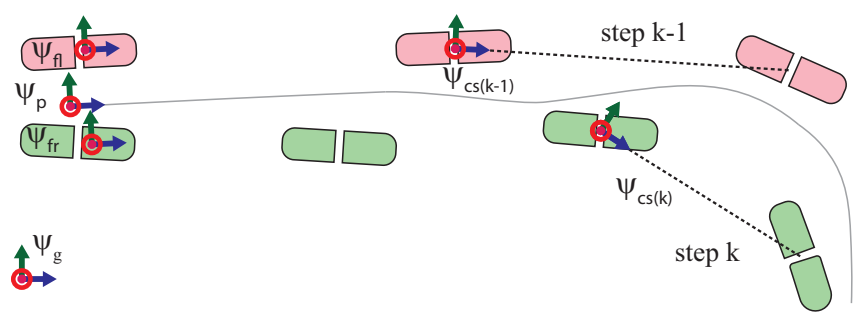

Fig. 1. Graphical interpretation of the frames [5]: The left foot is in pink, and the CoM trajectory is the thin gray line. A current step frame $\psi_{c s(k)}$ is employed for step $k$, instead of a fixed global frame $\psi_{g}$

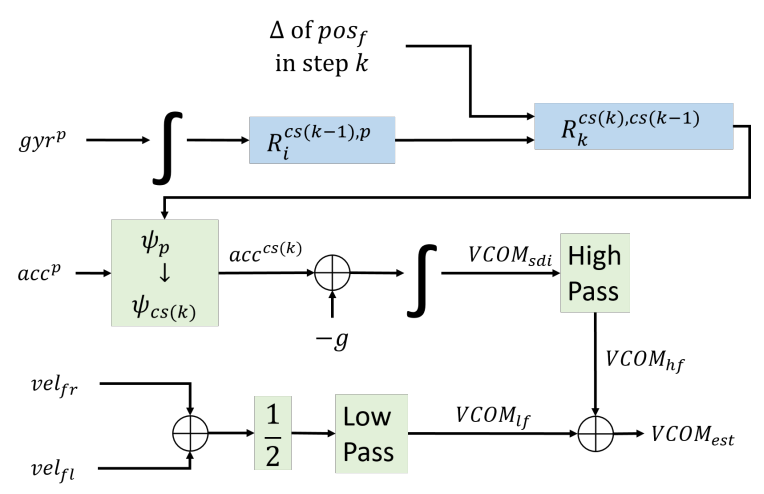

Fig. 2. Estimating instantaneous $\mathrm{CoM}$ velocity $\left(V C O M_{e s t}\right)$ : Pelvis accelerations $\left(a c c^{P}\right)$ are integrated after removing gravity, and high pass filtered to obtain $V C O M_{h f}$. This is fused with a low pass filtered $\left(V C O M_{l f}\right)$ average velocity of the feet using a complementary filter method. Each variable is expressed in a special current step $\psi_{c s}$ frame.

measuring the CoM accelerations (ACOM). Following this, Section II-C explains the estimations of an average VCOM from foot velocities, and the fusion of the two information sources.

\section{A. Using a Current Step Frame $\psi_{c s}$}

In this study, instead of a fixed global frame, a changing reference frame was employed [5]. The body centric current step frame, $\psi_{c s}$, was defined using the change in foot positions per step [5]. A graphical depiction is shown in Fig. 1. Fig. 2 summarizes the estimation of $\mathbf{R}_{k}^{c s(k), c s(k-1)}$ for the pelvis IMU. The steps are explained in detail in Mohamed Refai et al. [5]. To transform from sensor frame to current step frame makes, first a calibration to segment frame is performed 


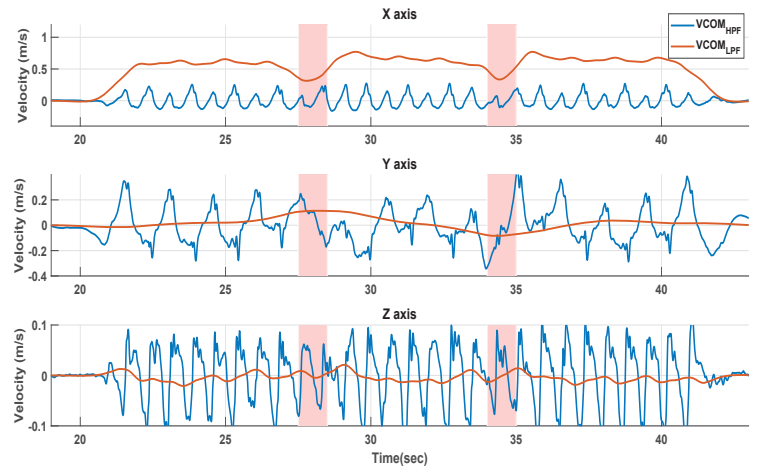

Fig. 3. Comparing the high frequency $V C O M_{h f}$ and low frequency $V C O M_{l f}$ for a WT2 task. Shaded regions denote turning moments.

[5], [6]. Then, an error extended Kalman filter (EEKF) was employed to track the changes in orientation during the step. At the end of the step, using the change in swing foot position in the horizontal floor plane as the heading, and $\mathrm{Z}$ axis along the vertical, a $\psi_{c s(k)}$ of the current step $k$ was defined.

\section{B. CoM Velocity using strapdown integration}

As seen in Fig. 2, gravity was removed from ACOM in $\psi_{c s(k)}\left(a c c^{c s(k)}\right)$, and then strapdown integrated using the direct and reverse integration method (DRI) [7] to obtain velocity $V C O M_{s d i}$. The velocities at the beginning and end of trial were set to 0 , as required by the DRI method. $V C O M_{s d i}$ is a time varying velocity estimate with drift that accumulates over time. Therefore, a high pass 2 nd order zero phase Butterworth filter was applied to obtain the $V C O M_{h f}$.

\section{CoM Velocity from foot velocities}

A low frequency estimate of the VCOM can be approximated from averaging the foot velocities. Drift free foot velocity estimates were obtained using an extended Kalman Filter and zero velocity constraints [8]. As seen in Fig. 2, the velocities of both feet $\left(v e l_{f l}\right.$ and $\left.v e l_{f r}\right)$ were averaged. A low pass 2nd order zero phase Butterworth filter was applied to obtain the $V C O M_{l f}$.

In order to employ a complementary filter [4], the cut off frequencies used for the high pass filter of $V C O M_{h f}$ and low pass filter of $V C O M_{l f}$ were the same. After a preliminary analysis, the optimal values were found to be $0.5,0.2$, and $1.4 \mathrm{~Hz}$ for $\mathrm{X}, \mathrm{Y}$, and $\mathrm{Z}$ axes respectively. The $V C O M_{h f}$ and $V C O M_{l f}$ were then fused to obtain the instantaneous VCOM $M_{\text {est }}$.

\section{Measurement System and Participants}

Three IMUs were used: One Xsens ${ }^{\mathrm{TM}}$ IMU was mounted on the sacrum using a strap, and one was placed on each foot on the midfoot region [5]. A MT Manager was used to read the data from the IMU wirelessly, which was sampled at $100 \mathrm{~Hz}$. A $\mathrm{VICON}^{\mathrm{C}}$ (Oxford Metrics PLC.) motion capture system was used as the reference. Markers were placed on the following locations on both the left and right limbs: anterior superior iliac spine, posterior iliac spine, the second and fifth metatarsal, and heel. The VICON ${ }^{\complement}$ was sampled at $100 \mathrm{~Hz}$.

The CoM position obtained from $\mathrm{VICON}{ }^{\complement}\left(P_{C O} M_{r e f}\right)$ was assumed to lie at the centroid of the pelvis markers. The $P C O M_{r e f}$ was differentiated and low pass filtered with a 2nd order zero phase Butterworth filter with cut off $10 \mathrm{~Hz}$ to obtain the VCOM ref. These were transformed to the $\psi_{c s(k)}$, determined independently using the foot position data of the $\mathrm{VICON}^{(}$.

Walking data was collected from trials by three healthy males. The mean height, weight, and age was $1.74 \pm 3 \mathrm{~m}$, $79.3 \pm 9 \mathrm{~kg}$, and $25 \pm 3.5$ years respectively. Leg length was $94 \pm 3 \mathrm{~cm}$ [9]. All participants signed an informed consent before the experiment. The study was conducted in accordance with the Declaration of Helsinki, and the protocol was approved by the Ethical Committee of the faculty.

\section{E. Experimental Protocol}

The subjects began by standing still for a few seconds, following which they were asked to bend the trunk forward thrice. Once the researcher gave the start sign, the subject walked along a given path. The following walking tasks were each repeated four times [10]:

1) Normal Walk $(N W)$ : The subject was asked to walk at their preferred walking speed for $5 \mathrm{~m}$.

2) $L$ Walk $(L W)$ : The subject was asked to walk for $3 \mathrm{~m}$ and then turn right at $90^{\circ}$ and walk for another $2 \mathrm{~m}$.

3) Walk and Turn (WT): The subject was asked to walk for $5 \mathrm{~m}$ and then turn and walk back to start position.

4) Walk and Turn Twice (WT2): The subject performed WT and then asked to turn and walk for $5 \mathrm{~m}$.

5) Slalom Walk $(S W)$ : The subject was asked to walk in a slalom pattern. Two pylons, at $2 \mathrm{~m}$ and $4 \mathrm{~m}$ from start respectively, were placed on the floor to guide them.

6) Asymmetric Walk $(A W)$ : The subject was asked to walk in an asymmetric manner. The instruction given was to induce a stiff left knee and abduct the hip as much as possible, and also have a shorter step on the right side.

\section{RESULTS}

Fig. 3 shows an example of $V C O M_{h f}$ (blue line) and $V C O M_{l f}$ (red line) for the WT2 task. The plot includes gait initiation, turning and termination. Following this, Fig. 4 shows an example of the estimated instantaneous $V C O M_{\text {est }}$ (blue line) compared with the reference $V C O M_{\text {ref }}$ (red line). Here, we also depict the strapdown integrated $V C O M_{s d i}$ (thin black line), which is seen to clearly drift. In both Fig. 3 and 4 , the subject makes two $180^{\circ}$ turns which are denoted by shaded light red rectangles. Also, in both figures, each subplot corresponds to an axis of the $\psi_{c s(k)}$. Table I compares the average root mean square of the error between the $V C O M_{e s t}$ and $V C O M_{r e f}$ across all subjects for different walking tasks as both absolute, and percentage error normalized to the range of $V C O M_{\text {ref }}$. 


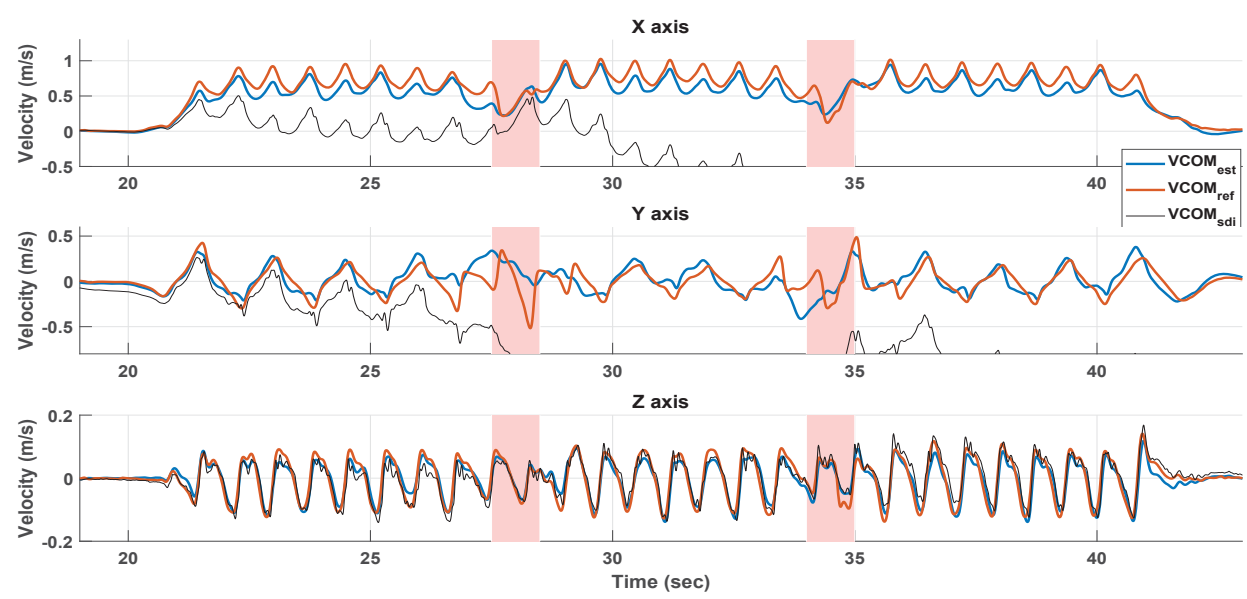

Fig. 4. Comparing the estimated $V C O M_{e s t}$, and $V C O M_{s d i}$, and $V C O M_{r e f}$ for a WT2 task. Shaded regions denote turning moments.

TABLE I

AbSOlute AND PERCENTAGE ROOT MEAN SQUARE ERROR BETWEen $V C O M_{e s t}$ AND $V C O M_{r e f}$

\begin{tabular}{l|cc|cc|cc}
\hline & $\mathbf{R M S}_{\mathbf{X}}(\mathrm{m} / \mathrm{s})$ & $\mathbf{R M S}_{\mathbf{X}}(\%)$ & $\mathbf{R M S}_{\mathbf{Y}}(\mathrm{m} / \mathrm{s})$ & $\mathbf{R M S}_{\mathbf{Y}}(\%)$ & $\mathbf{R M S}_{\mathbf{Z}}(\mathrm{m} / \mathrm{s})$ & $\mathbf{R M S}_{\mathbf{Z}}(\%)$ \\
\hline $\mathbf{N W}$ & $0.1 \pm 0.03$ & $12.2 \pm 3.1$ & $0.1 \pm 0.02$ & $13.9 \pm 4.2$ & $0.1 \pm 0.01$ & $11.6 \pm 5.3$ \\
\hline $\mathbf{L W}$ & $0.1 \pm 0.01$ & $9.0 \pm 1.4$ & $0.2 \pm 0.05$ & $15.0 \pm 2.6$ & $0.1 \pm 0.01$ & $12.0 \pm 2.8$ \\
\hline $\mathbf{W T}$ & $0.1 \pm 0.04$ & $10.1 \pm 2.6$ & $0.2 \pm 0.02$ & $14.4 \pm 2.3$ & $0.1 \pm 0.01$ & $12.3 \pm 3.0$ \\
\hline $\mathbf{W T 2}$ & $0.2 \pm 0.04$ & $11.5 \pm 1.8$ & $0.2 \pm 0.07$ & $11.9 \pm 2.0$ & $0.1 \pm 0.02$ & $12.1 \pm 2.8$ \\
\hline $\mathbf{S I W}$ & $0.1 \pm 0.02$ & $12.8 \pm 3.5$ & $0.2 \pm 0.01$ & $18.6 \pm 1.3$ & $0.1 \pm 0.01$ & $18.9 \pm 6.1$ \\
\hline $\mathbf{A W}$ & $0.1 \pm 0.02$ & $12.2 \pm 6.7$ & $0.1 \pm 0.02$ & $13.4 \pm 3.3$ & $0.1 \pm 0.02$ & $15.7 \pm 2.2$ \\
\hline
\end{tabular}

NW: Normal Walk, LW: L Walk, WT: Walk and Turn, WT2: Walk and Turn Twice, SlW: Slalom Walk, AW: Asymmetrical Walk.

\section{Discussion}

The current method shows the feasibility of estimating 3D VCOM during variable gait. Although, the complementary method is similar to Sabatini et al. [3], they used machine learning to estimate the average VCOM. Our approach discards the need for a training step by including the foot IMUs. Note that the use of foot IMUs is two-fold: for defining the $\psi_{c s(k)}$ as well as obtaining a low frequency VCOM information. Fig. 3 shows the complementary information present in the $V C O M_{h f}$ and $V C O M_{l f}$. VCOM $M_{l f}$ derived from the foot velocities encodes the trend and $V C O M_{h f}$ has information regarding a drift free change in this trend. In Fig. 3 and 4, the kinematics are expressed in $\psi_{c s}$, and hence, the velocities remain positive in the $\mathrm{X}$ axis even as the subject makes two $180^{\circ}$ turns, during the shaded regions. Note that in Fig. 4, the drift in the vertical $V C O M_{s d i}$ is quite limited compared to the other axes, but exists, as evident during the last few steps. Further, the $V C O M_{\text {ref }}$ shows more drastic jumps during the turns, as compared to $V C O M_{e s t}$, more clearly seen in the $\mathrm{Y}$ axis due to transformation to the $\psi_{c s}$. As we account for the $R_{k}^{c s(k), c s(k-1)}$ per step $k$, we can represent the kinematics in a fixed global frame, or the frame of any other required step.

Table I shows that the errors are on average $13.1 \pm 2.2 \%$ of the range of VCOM across all axes and walking tasks. The errors seem to be largest for the SIW task, as the gait was always changing direction. The error margins are quite low overall, about less than $19 \%$ of the range in the worst case. The algorithm has lower errors for variable walking when compared to the results of Sabatini et al. [3]. The applicability of the method however, would dependent on the application, and proposed error margins. Note that the cut offs used in the complementary filter was optimized across all subjects. The errors found could be further lowered if this was optimized per subject. A drawback of this method is that it employs a DRI method for integration, which requires knowledge of the final state of the velocities.

A three IMU setup can provide a minimal sensing setup for drift free estimates of CoM velocity. This can further improve estimates of the position of COM, and XCOM.

\section{ACKNOWLEDGMENT}

This work is part of the Perspectief programme NeuroCIMT with project number 14905 which is (partly) financed by the Netherlands Organisation for Scientific Research (NWO). 


\section{REFERENCES}

[1] F. B. van Meulen, D. Weenk, E. H. F. van Asseldonk, H. M. Schepers, P. H. Veltink, and J. H. Buurke, "Analysis of balance during functional walking in stroke survivors," Plos One, vol. 11, no. 11, p. 20, 2016. [Online]. Available: http://dx.plos.org/10.1371/journal.pone.0166789

[2] A. Mannini and A. M. Sabatini, "Walking speed estimation using foot-mounted inertial sensors: Comparing machine learning and strap-down integration methods," Medical Engineering and Physics, vol. 36, no. 10, pp. 1312-1321, 2014. [Online]. Available: http://dx.doi.org/10.1016/j.medengphy.2014.07.022

[3] A. M. Sabatini and A. Mannini, "Ambulatory Assessment of Instantaneous Velocity during Walking Using Inertial Sensor Measurements," Sensors, vol. 16, no. 12, p. 2206, dec 2016. [Online]. Available: http://www.mdpi.com/1424-8220/16/12/2206

[4] H. M. Schepers, E. H. F. van Asseldonk, J. H. Buurke, and P. H. Veltink, "Ambulatory Estimation of Center of Mass Displacement During Walking," IEEE Transactions on Biomedical Engineering, vol. 56, no. 4, pp. 1189-1195, 2009. [Online]. Available: https://ieeexplore.ieee.org/document/4760226

[5] M. I. Mohamed Refai, B.-J. F. Van Beijnum, J. H. Buurke, and P. H. Veltink, "Portable Gait Lab: Estimating 3D GRF using a Pelvis IMU in a foot IMU defined frame," IEEE transactions on neural systems and rehabilitation engineering, April 2020. [Online]. Available: https://doi.org/10.1109/TNSRE.2020.2984809

[6] S. Bonnet, C. Bassompierre, C. Godin, S. Lesecq, and A. Barraud, "Calibration methods for inertial and magnetic sensors," Sensors and Actuators, A: Physical, vol. 156, no. 2, pp. 302-311, 2009.

[7] M. Zok, C. Mazzà, and U. Della Croce, "Total body centre of mass displacement estimated using ground reactions during transitory motor tasks: application to step ascent," Medical Engineering \& Physics, vol. 26, no. 9, pp. 791-798, nov 2004.

[8] D. Weenk, D. Roetenberg, B.-J. F. van Beijnum, H. Hermens, and P. H. Veltink, "Ambulatory estimation of relative foot positions by fusing ultrasound and inertial sensor data," IEEE Transactions on Neural Systems and Rehabilitation Engineering, vol. 23, no. 5, pp. 817-826, 2015. [Online]. Available: https://ieeexplore.ieee.org/document/6898866/

[9] A. L. Hof, "Scaling gait data to body size," Gait and Posture, vol. 4, no. 3, pp. 222-223, 1996

[10] M. I. Mohamed Refai, B.-J. F. Van Beijnum, J. H. Buurke, and P. H. Veltink, "Portable gait lab: Tracking relative distances of feet and com using three imus," IEEE Transactions on Neural Systems and Rehabilitation Engineering, 2020, preprint. [Online]. Available: https://ieeexplore.ieee.org/document/9171861 